\title{
Participación activa de las y los estudiantes en el diseño de su aprendizaje. Una experiencia educativa en Trabajo Social
}

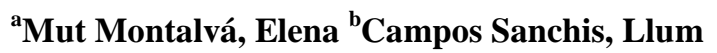 \\ ${ }^{a}$ Universitat de València, España, elena.mut@uv.es ${ }^{\mathrm{b}}$ Universitat de València, España, \\ m.llum.campos@uv.es
}

\begin{abstract}
Resumen
Se presenta una reflexión teórico-práctica sobre experiencias docentes metodológicas y de aprendizaje en el Grado en Trabajo Social de la Universitat de València basadas en las teorías del aprendizaje significativo de Ausebel, del aprendizaje social de Bandura o Vigosky y las propuestas de intervención Social de Ander-Egg y De Robertis, comunidades de aprendizaje, Aprendizaje Servicio e Investigación-Acción Participativa. En las conclusiones se presentan algunas implicaciones para la docencia universitaria y para futuras investigaciones.
\end{abstract}

Palabras clave: innovación docente, aprendizaje significativo, metodología docente universitaria, competencias de aprendizaje

\section{Introducción}

Los cambios producidos en el contexto del EEES (Espacio Europeo de Educación Superior) se han dirigido hacia un proceso de enseñanza-aprendizaje cuyo centro de atención es el alumnado. En este sentido, dicho proceso constructivista se ha orientado a las percepciones, preferencias y concepciones del alumnado sobre la enseñanza y el aprendizaje. En el caso de la disciplina del Trabajo Social, de ella deriva una actividad profesional que tiene por objeto el diagnóstico, la intervención y la evaluación social de las necesidades sociales para promover la resolución de los problemas en las relaciones humanas, el fortalecimiento democrático y la promoción del cambio social para el logro de una sociedad inclusiva y cohesionada. Todo ello hace necesario considerar el contexto social, económico, tecnológico y cultural en el cual se inscribe el Trabajo Social, pues las características de dichos contextos tan diversos deben aplicarse en la formación de las y los futuros profesionales del trabajo social. 
Participación activa de las y los estudiantes en el diseño de su aprendizaje. Una experiencia educativa en Trabajo Social.

Los perfiles de dicha figura profesional están influidos por un conjunto de factores que presentan una relación dialéctica con la sociedad. Por ello, resulta imprescindible incorporar las aportaciones procedentes de las nuevas demandas sociales y de las dinámicas demográficas actuales, favoreciendo una profundización de la reflexión sobre la propia práctica.

El presente estudio analiza y evalua la experiencia docente de las autoras en cuatro asignaturas de los cursos de segundo, tercero y cuarto del Grado en Trabajo Social de la Universitat de València y que son: Proyectos Sociales, Comunicación Profesional, Trabajo Social con personas con Diversidad Funcional y Trabajo Social en Diferentes Contextos Educativos. Cabe destacar que en todas ellas se incorpora, además y de forma transversal, la perspectiva de género.

La formación académica de las y los trabajadores sociales comprende una dimensión integral que atiende a contenidos disciplinares, competenciales y actitudinales que contribuyen a generar profesionales que no solo sepan hacer, sino que también conozcan la diversidad sociocultural y étnica del contexto en el que van a desempeñarse profesionalemnte (tendrán que tratar con grupos diversos) para que sean capaces de analizar críticamente la realidad e intervenir para disminuir las discriminaciones y las desigualdades socioeconómicas.

\section{Aproximación metodológica y teórica}

La forma en cómo accedemos al conocimiento en las Ciencias Sociales es circular, cada conocimiento se nos presenta de una manera y volvemos a él desde diferentes perspectivas y posiciones, de forma que nunca está cerrado o acabado. Este rasgo característico de las Ciencias Sociales otorga a nuestro trabajo como docentes las dificultades añadidas de la secuenciación de unos contenidos que, en la realidad, se dan todos a la vez, en una o varias dimensiones, pero en un mismo contexto temporal. Una vez somos conscientes de esta premisa epistemológica, tenemos la libertad de articular todos estos conocimientos desde ópticas diferentes y, en función de realidades también diferentes, se adapta el proceso de enseñanza-aprendizaje a un contexto social teniendo en cuenta el grupo, el aula y la materia a impartir.

La experiencia docente a lo largo de siete cursos académicos desde la puesta en marcha del plan de estudios del Grado nos ha permitido identificar qué se va a aprender (además de lo que se describe en las guias docentes de cada una de las diferentes asignaturas) y la pertinencia de que el alumnado participe en el cómo se va a aprender, siendo éste el 
protagonista y responsable de su propio aprendizaje. Para ello, comparten sus vivencias y recursos personales y sociales, así como identifican y localizan aquellos recursos disponibles en su entorno social más próximo, compartiéndolos, a su vez, con otros estudiantes. Por ello, resulta imperativo flexibilizar la metodología de enseñanza, pues está en permanente conexión con el contexto y permite que las situaciones que el alumnado experimenta y percibe puedan ser aprovechadas como potencialidades y oportunidades para enriquecer el proceso de enseñanza-aprendizaje.

El objeto de estudio de la investigación ha consistido en el estudio sobre los beneficios en el aprendizaje para los y las estudiantes del Grado en Trabajo Social de la Universitatd de Valencia a través de la metodología significativa-social para la asimilación de contenidos. En dicha investigación se ha utilizado una metodología de carácter cualitativo en la que se han aplicado las siguientes técnicas: el análisis documental y bibliográfico sobre pedagogías de aprendizaje; la observación participante realizada en las aulas y en las actividades conexas (jornadas, reuniones) y el análisis de los instrumentos de registro y evaluación aplicados a los y las estudiantes a lo largo de siete cursos académicos en el Grado en Trabajo Social. El periodo de estudio inicia en el curso 2012/13 y finaliza en el curso 2018/19, con una muestra de 278 estudiantes.

Este proceso esta basado en las aportaciones de diversos autores y autoras que abarcan tanto pedagogías tradicionales como contemporáneas, y que se combinan con las teorías del aprendizaje significativo de Ausubel y del aprendizaje social de Alfred Bandura o de Vigosky. También incorpora las propuestas metodológicas de intervención Social de Ezequiel Ander-Egg o Cristina de Robertis y otras metodologías como: Comunidades de Aprendizaje, Aprendizaje y Servicio e Investigación Acción Participativa.

Partimos de la premisa de que el alumnado experimenta una serie de vivencias y conocimientos que afectan su aprendizaje y que, además, pueden ser aprovechados para su beneficio. Ausubel resume este hecho de la siguiente manera: "Si tuviese que reducir toda la psicología educativa a un solo principio, enunciaría este: El factor más importante que influye en el aprendizaje es lo que el alumno ya sabe. Averígüese esto y enséñese consecuentemente" (1983:2). Por otro lado, Vygotsky afirmó que "el aprendizaje activa una serie de procesos internos de desarrollo que son capaces de operar sólo cuando el alumno/a está interactuando con personas de su entorno y en cooperación con sus compañeros" (1979: 89).

Las anteriores aportaciones teóricas han cristalizado en la metodología aplicada que aunaría las teorías de estos dos autores y que nosotras la hemos denominado "metodologia significativa-social". A modo de ejemplo, en la asignatura de Proyectos Sociales de $3^{\circ}$ curso, mediante el aprendizaje significativo logramos que el alumnado se involucre en el proceso de aprendizaje haciéndoles responsables del mismo; que observe y vigile su 
Participación activa de las y los estudiantes en el diseño de su aprendizaje. Una experiencia educativa en Trabajo Social.

entorno; que obtenga información actualizada y la aporte al aula (revisión de prensa y de otros medios de comunicación); que comparta su experiencia y recursos personales y que tenga la oportunidad de participar en eventos y actividades de su entorno sociocultural que puedan estar relacionados con los temarios que se están tratando en el aula. En este caso, el trabajo obligatorio a realizar para superar la asignatura consiste en realizar un Projecto de Intervención Social. Para ello, desde la primera sesion se crean los grupos de trabajo para facilitar que compartan sus propios recursos personales y sociales, que localicen los que tienen en su entorno social más próximo (y que hasta el momento eran invisibles o pasaban desapercibidos, quizá por que no los habían nombrado o no se habían parado a pensar por que no los habían necesitado). El aula se convierte en un espacio de reflexión que posibilita el pensamiento crítico y creativo. Este fenómeno no se da de manera espontánea, pues hay que posibilitarlo dentro del aula para descubrir al alumnado los beneficios que a las personas nos supone tener información. Todo este proceso está muy relacionado con la metodologia Investigación-Acción-Participativa.

Tomas Villasante destaca que uno de los principios freirianos acerca de que nadie puede educar a nadie, ni siquiera a sí mismo, ha de darse en el aprendizaje común mediatizados por el mundo. Es decir, construir espacios creativos en donde todas las personas podamos ir aprendiendo, desde la experiencia que cada cual traiga (Villasante, 2000 y 2008).

\section{Resultados}

La metodologia significativa-social aplicada en las aulas ha desarrollado un enfoque participativo y experiencial y, por tanto, las y los estudiantes han estado en contacto constante con las diversas realidades de su entorno territorial y con las diversidades socioculturales y étnicas de su entorno más próximo. Para promover dicha participación se ha contado con la implicación del propio alumnado en la organización de las sesiones docentes. Por otro lado, también ha sido necesario conocer previamente sus potencialidades, sus recursos y experiencias sociales, es decir, es importante saber quiénes son los y las estudiantes universitarios como sujetos y objetos en su eco-territorio. 


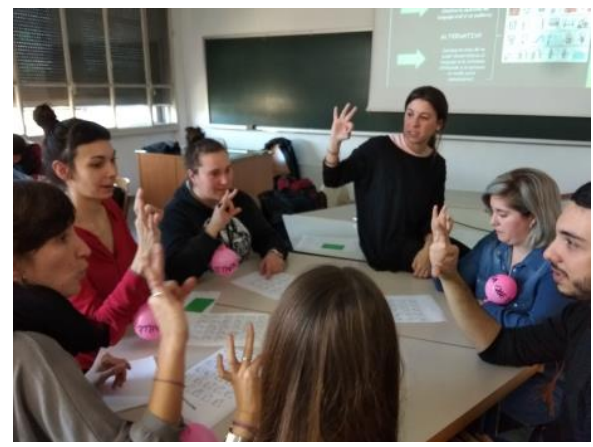

Fig.1 Alumnado de la asignatura Trabajo Social con personas con Diversidad Funcional. Sesión Lenguaje de signos impartida por una alumna del grupo. Curso 2018/19

Fuente: Llum Campos

En cuanto a las cuestiones metodológicas se utilizan y combinan diferentes estrategias, en función del tipo de actividad, de la secuenciación de la misma, de los contenidos implicados y de los resultados de aprendizaje a obtener. Por ello, la intervención teórica y de exposición por parte del profesorado va a ser mínima en las primeras sesiones. Lo más idóneo es utilizar una metodología grupal y participativa, significativa y experiencial, tanto fuera como dentro del aula. De esta forma, se consigue fomentar las vivencias y experiencias sobre los diversos contenidos de las asignaturas por lo que salir de las aulas y encontrar esa parte significativa que da sentido al aprendizaje de dichos contenidos teóricos y su asimilación posibilita, a su vez, que los conocimientos adquiridos sean más estables y duraderos.

El proceso de aprendizaje se completa con la consulta, estudio de textos y análisis documental (artículos científicos, informes y estudios de casos) que promueven y favorecen una reflexión situada sobre los conceptos estudiados y las perspectivas en el ámbito del desarrollo comunitario y social, así como de las técnicas de investigación aplicadas. De esta forma, se fomenta la creatividad y la aplicación y uso de las nuevas tecnologías, pues está constatado que entre las y los estudiantes el uso de las redes sociales es un elemento fundamental de socialización y comunicación, y no es posible obviar esta realidad.

Como ya se ha indicado, en la mayoría de las sesiones las y los estudiantes se organizan en grupos mixtos (para incorporar la diversidad sexual existente en cada aula), para después realizar una exposición con las conclusiones sobre la temática realizada al gran grupo, o bien, fomentar debates participativos. El alumnado experimenta en el aula una dinámica y forma de trabajar similar a la que sería ideal encontrar en su futuro profesional en el ámbito del trabajo social, donde trabajará como miembro de un equipo interdisciplinar más amplio y con una diversidad de grupos humanos. Como se ha descrito en la introducción, ello está 
Participación activa de las y los estudiantes en el diseño de su aprendizaje. Una experiencia educativa en Trabajo Social.

en consonancia con los perfiles de la figura profesional que está en constate dialéctica con la sociedad.

Adicionalmente, se fomenta el uso de una metodología participativa que pretende que las y los estudiantes participen de manera activa en el aula. Se estimula su participación para realizar aportaciones y favorecer el diálogo entre iguales y se establecen debates. Todo ello hace imprescindible realizar previamente sesiones para que el alumnado "aprenda a aprender", realizando diferentes técnicas y dinámicas de grupo que, además, son estrategias claves en la formación de los y las futuros profesionales del Trabajo Social.

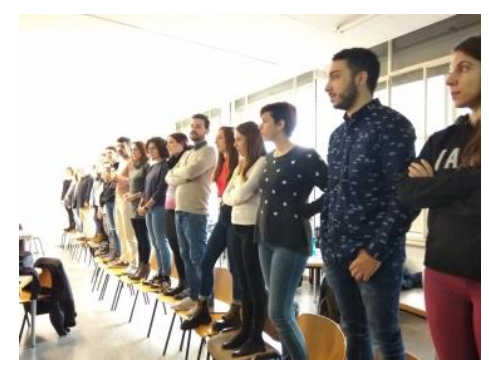

Fig. 2 Alumnado de la asignatura Proyectos Sociales. Sesión de cohesión grupal. Fuente: Llum Campos

Nuestro alumnado se incorpora a la universidad con la competencia adquirida para aprender a aprender, ya que es una de las competencia clave de la LOMCE donde se afirma que aprender a aprender es fundamental para el aprendizaje permanente que se produce a lo largo de la vida y que tiene lugar en distintos contextos formales, no formales e informales. Supone la habilidad para iniciar, organizar y persistir en el aprendizaje. Respecto a las actitudes y valores, la motivación y la confianza son cruciales para la adquisición de esta competencia. Ambas se potencian desde el planteamiento de metas realistas a corto, medio y largo plazo. Al alcanzarse las metas aumenta la percepción de auto-eficacia y la confianza, y con ello se elevan los objetivos de aprendizaje de forma progresiva. Las personas deben ser capaces de apoyarse en experiencias vitales y de aprendizaje previas con el fin de utilizar y aplicar los nuevos conocimientos y capacidades en otros contextos, como los de la vida privada y profesional, la educación y la formación (LOMCE, 2013).

Desde la perspectiva de las y los docentes es necesario destacar el enorme esfuerzo que supone la tarea de diseño, planificación, realización, coordinación con otras asignaturas y evaluación para aplicar con rigor dicha propuesta metodológica de aprendizaje, la cual requiere la interrelación y conectividad de los contenidos de las propias asignaturas con los procedimientos metodológicos para alcanzar los resultados de aprendizaje de cada materia y evaluarlas adecuadamente. En este sentido, se proporciona al estundiantado instrumentos de registro que ayudan tanto a la evaluación docente, como a la autoevaluación de manera 
continua y asi poder modificar aquellas dinámicas de trabajo que no estén resultando productivas o positivas para el grupo. Se ha diseñado un acta para registrar en cada sesión el funcionamiento del grupo en el espacio de trabajo, su composición y dinámica. El alumnado recoge en ella información de manera clara, fácil de rellenar, se genera reflexión y debate y extraen sus propias conclusiones. Se registra a las personas que han participado en cada sesión y el cumplimiento de objetivos, lo que plantea repensar la operatividad y resultados de los temas abordados y los acuerdos alcanzados, la propuesta de trabajo para la próxima sesión, así como la dinámica en general del equipo.

Dependiendo de la materia, se utilizan también como instrumentos de registro básicos fichas de registro de las actividades realizadas y una ficha de entidades, que diseñarán según las necesidades cada grupo, se proporcionan algunos ejemplos pero han de adaptar y crear sus propias fichas donde recogeran los datos pertinentes para cada caso. En ella incluyen además de los datos generales de la entidad un apartado con las observaciones de información que esta entidad a proporcionado a su trabajo, como se ha establecido el contacto, el proceso y la dinámica de la colaboración generada, así como la evaluación de la experiencia.

Los resultaos de aprendizaje consiste en que: el alumnado aprende a sistematizar el trabajo a través de la utilización de los diferentes instrumentos de registro planteados y aprende a definir qué información es relevante. Por otro lado, para el profesorado aporta información cualitativa sobre sus motivaciones para hacer la actividad, el crecimiento personal y la evolución hacia lo profesional en una secuencia desde la primera a la última acta, así como de las expectativas previas a la experiencia vivida en el aula y sus valoraciones respecto al resultado final. También aporta información que nos ayuda a evaluar y analizar los vínculos y las relaciones establecidas antes y después. Con esta información diseñamos indicadores para la evaluación final de su trabajo y de la propia materia impartida y se realizan conjuntamente propuestas de mejora que se tendran en cuenta para el futuro.

En la aplicación de esta metodología se es conciente de que no todas las materias del Grado permiten el uso de la metodología planteada, sobre todo aquellas que requieren de unos conocimientos previos teóricos, históricos o de conocimiento legislativo entre otros. A partir de transmitir al alumnado la base del conocimiento científico-teórico es más fácil participar. Si el estudiantado cuenta con una información de partida la puede ampliar, sistematizar, comparar, abstraer y alcanzar sus propias conclusiones para las materias más practicas donde se aplica la metodología propuesta.

De forma sintética, los principales resultados obtenidos tras la aplicación de las técnicas de investigación descritas han sido los siguientes:

Se ha constatado la significativa motivación del alumnado por la experiencia vivencial obtenida en cada una de las sesiones. En su gran mayoría, han valorado positivamente la 
Participación activa de las y los estudiantes en el diseño de su aprendizaje. Una experiencia educativa en Trabajo Social.

forma en la que han adquirido el conocimiento. A diferencia de otras metodologías, en la que se ha estudiado se han adquirido los contenidos de forma compacta, cada elemento está relacionado con otros, como en una gran red o sistema. En concreto, los estudiantes obtienen un gran mapa conceptual relacionado, más que una serie lineal y estática, porque la realidad social no es lineal ni estática, sino multiforme y dinámica.

Los resultados indican que es más importante considerar los contenidos procedimentales referidos a cómo han asimilado la metodología propuesta y, al mismo nivel, las actitudes que el alumnado ha adquirido en su proceso de maduración y evolución personal y académica. En este sentido, los resultados de aprendizaje son muy positivos, pues se ha demostrado el logro, en un porcentaje elevado (96\% de los 278 estudiantes analizados), de adquisición de las competencias generales contempladas en el plan de estudios del grado, y que son básicas para devenir un buen profesional del Trabajo Social: 1) Capacidad para trabajar y valorar de manera conjunta con personas, familias, grupos, organizaciones y comunidades sus necesidades y sus circunstancias. 2) Capacidad para planificar, implementar, revisar y evaluar la práctica del Trabajo Social con personas, familias, grupos, organizaciones, comunidades y con otros y otra profesionales. 3) Capacidad para apoyar a las personas para que sean capaces de manifestar las necesidades, puntos de vista y circunstancias. 4) Capacidad para actuar en la resolución de las situaciones de riesgo con las personas así como para las propias y las de las y los colegas de la profesión. 5) Capacidad para administrar y ser responsable, con supervisión y apoyo de la propia práctica dentro de la organización y, por último, 6) Capacidad para demostrar competencia profesional en el ejercicio del Trabajo Social.

Todas estas competencias generales están vinculadas a la competencia transversal y a todas las materias del título y que es la relativa a la capacidad para transmitir y potenciar la igualdad de oportunidades, la accesibilidad universal a los derechos humanos de mujeres y hombres, los valores de democracia y paz y la sostenibilidad. (Memoria de verificación del título oficial de Grado: Graduado en Trabajo Social, Universidad de Valencia, 2011).

\section{Conclusiones}

Las conclusiones de la investigación que se presenta tienen la consideración de aproximaciones al objeto de estudio, pues la obtención de conclusiones de mayor alcance requeriría de la utilización de un mayor número de técnicas de investigación y de la ampliación de la muestra. Aún así, los resultados de esta investigación demuestran la pertinencia de seguir impulsando este tipo de metodologías para poder avanzar en la mejora del proceso de enseñanza-aprendizaje y contribuir, además, al desarrollo de habilidades y 
capacidades en el alumnado para su futuro desarrollo profesional en una socirdad cambiante y diversa. La adquisición de éstas favorece su contribución a la transformación social y a la reducción de las desigualdades, siendo capaces de transferir lo aprendido al campo profesional donde seguirán sumando experiencias que favorezcan la reorganización de su pensamiento, la adaptación de sus actuaciones y la incorporación de otras habilidades para ejecutar eficientemente sus intervenciones. Es decir, la adquisión de capacidades y habilidades para un aprendizaje continuo a lo largo de toda la vida.

La neuroeducación, como aplicación de la neurociencia en el ámbito de las aulas, nos dice que el gran cambio está en las emociones, en cómo el alumnado vive y experimenta su proceso de aprendizaje. La metodología propuesta motiva a las y los estudiantes al trabajo en el aula, pero también al profesorado al comprobar que somos responsables de generar emociones positivas en este espacio de aprendizaje, obteniendo así un aula con un ambiente colaborativo y donde el alumnado muestra una respuesta positiva ante la propuesta metodológica de trabajo donde ellos y ellas son, a su vez, responsables de crear un ambiente de motivación y de trabajo donde se valora su autoaprendizaje. De esta forma, el profesorado nos sorprendemos, aprendemos del alumnado y mostramos interés, nos motivamos orientándoles en el proceso pero, a la vez, aceptando sus propuestas e ideas lo que favorece procesos de empoderamiento y de generación de la seguridad en la toma de decisiones, que constiyuyen características básicas para su futuro profesional y personal.

Consideramos que nuestra profesión, como docentes de educación superior, impacta en la comunidad estudiantil universitaria en dos vías: por un lado, en la formación de alta calidad de las y los futuros profesionales del Trabajo Social o investigadores sociales. Y, por otro lado, en la generación de estudiantes dotados de una conciencia crítica y comprometidos con su entorno y, por tanto, con los valores de solidaridad, cooperación y justicia social. Forma parte de los principios éticos y morales contribuir a la mejor formación profesional de los y las estudiantes para que puedan impulsar los cambios necesarios para conformar sociedades más justas, inclusivas y democráticas.

\section{Referencias}

Ander-Egg, E. (2003). Planificación Educativa. Barcelona: Editorial Lumen Distribuidora S.R.L

Ausubel, D.P. (1983). Teoría del Aprendizaje significativo. Psicolgía educativa. Mejico: Trillas.

Ausubel, D.P. (2002) Adqusición y retención del conocimiento.Una perspectiva cognitiva. Barcelona: Paidós.

Barandiaran, M. (2013). El concepto de calidad en educación superior, una estrategia de apoyo desde las universidades a la generación del Desarrollo Humano (Tesis Doctoral). http://www.hegoa.ehu.es/es/articles/text/nueva tesis doctoral en hegoa33333 
Participación activa de las y los estudiantes en el diseño de su aprendizaje. Una experiencia educativa en Trabajo Social.

Díez-Palomar, J \& Flecha García, R. (2010). Comunidades de Aprendizaje: un proyecto de transformación social y educativa. Revista Interuniversitaria de Formación del Profesorado, 67 $(24,1), 19-30$ http://www.aufop.com/aufop/revistas/arta/impresa/146/1357

Ley Orgánica 8/2013, de 9 de diciembre, para la mejora de la calidad educativa (LOMCE). https://www.educacionyfp.gob.es/educacion/mc/lomce/inicio.html

Monclus, C. (2016). TIC, cooperació, diàleg i construcció de coneixements en Calvo, Cano El aprendizaje cooperativo como práctica docente: experiencias aplicadas. Alzira: Editorial Neopatria.

Universidad de Valencia. (2011). Memoria de verificación del título oficial de Grado: Graduado en Trabajo Social. https://www.uv.es/graus/verifica/Treball_Social/Memoria_desembre_2013.pdf

UNESCO (2015). Replantear la educación. ¿Hacia el bien común global? UNESCO: Paris.

Vygotsky, L.S. (1979). El desarrollo de los procesos psicológicos superiores. Barcelona: Crítica.

Villasante, T., \& Montañes, M., \& Martí, J. (2000). La investigación social participativa/Construyendo ciudadanía 1. España: El Viejo Topo.

Villasante, T. \& Martín, P. (2008). Constuyendo ciudadanía: proyecto "escuelas de ciudadanía solidaria". Cuadernos CIMAS. Observatorio Internacional de Ciudadanía y Medioambiente Sostenible. Ayuntamiento de Sevilla. 